\title{
Atención médica integral en pacientes con enfermedad hepática
}

\author{
Comprehensive medical care in patients with liver disease
}

\section{Assistência médica abrangente em pacientes com doença hepática}

\author{
Hernán D. Toro-Barrera ${ }^{\mathrm{I}}$ \\ hdavidtorob@gmail.com \\ Inés L. Molina-Saltos II \\ lorelu1406@hotmail.com \\ Hugo J. Saltos-Giler ${ }^{\text {III }}$ \\ javilifer@hotmail.com \\ Marcos A. Bedoya-Romo IV \\ marcos4786@hotmail.es \\ Pierina E. Saltos-Montes V \\ pierina_s.m@hotmail.com \\ Julio C. Cedeño-Quijije ${ }^{\mathrm{VI}}$ \\ jucecequi@hotmail.com
}

Recibido: 10 de enero de 2019 *Aceptado: 08 de febrero de 2019* Publicado: 05 de abril de 2019

\footnotetext{
Médico Cirujano; Médico Residente de UCI Clínica Centeno; Médico Residente de Emergencia y Jefe de Guardia Hospital General IESS; Manta, Ecuador.

Médico General; Médico Residente Asistencial; Hospital de Especialidades de las Fuerzas Armadas N`1; Quito, Ecuador.

Médico Cirujano; Médico Residente de Área de Cirugía Hospital General IESS; Manta, Ecuador.

Médico Cirujano; Médico Residente UCI del Hospital General IESS; Manta, Ecuador.

Médico Cirujano; Médico Residente del Hospital General IESS; Manta, Ecuador.

VI. Médico Cirujano; Médico Residente de Emergencia y Jefe de Guardia Hospital General IESS; Manta,Ecuador.
} 


\title{
Resumen
}

La atención médica integral representa el instrumento metodológico más efectivo para mejorar de manera constante la calidad de vida e incremento de los cuidados de los pacientes afectados con enfermedades hepáticas. Esta política sanitaria, responde a un método sistematizado y organizado dirigido a brindar cuidados, a través de la valoración, diagnóstico, planificación, intervención y evaluación de acuerdo a las respuestas humanas de la persona, utilizando el método científico como sustento de las acciones, lo que permite abordar un plan de cuidados, según sea la patología y gravedad del enfermo, considerándose para ello que, los pacientes con enfermedad hepática (enfermedades del hígado), crónicas, encontrándose entre ellas, la hepatitis A, B y C, Hígado Graso, Enfermedades Autoinmunes y la Cirrosis; esta última caracterizada por tener mayor riesgo de readmisión hospitalaria, hallándose una mortalidad de $13 \%$ a los 90 días, y que las causas más comunes de esta afección médica en países desarrollados son la hepatitis viral crónica tanto $\mathrm{B}$ o $\mathrm{C}$, hepatopatía alcohólica, hemocromatosis y hepatopatía no alcohólica, menos comunes son la hepatitis autoinmune, cirrosis biliar primaria y secundaria, colangitis esclerosante primaria, medicación como metotrexate e isoniazida, enfermedad de Wilson, deficiencia de alfa-1 antitripsina, padecimientos éstos que por su condición clínica, deben ser tratados bajo orientaciones médicas dirigidas por los diferentes programas y políticas en salud, los cuales tienen como propósito garantizar a las poblaciones (en este caso pacientes con enfermedades hepáticas) la continuidad de los cuidados garantizando sobre bases científicas, tanto la evaluación, como la aplicación del tratamiento adecuado; de ahí, la importancia que tienen estos servicios de atención sanitaria, los cuales buscan brindar a los pacientes con insuficiencia hepática crónica un diagnóstico oportuno y la identificación de factores precipitantes, lográndose de esa forma, mejorar el estado clínico de la persona.

Palabras claves: Atención Médica Integral; Políticas de Salud; Enfermedades Hepáticas; Cirrosis Hepática; Encefalopatía Hepática.

\begin{abstract}
Comprehensive medical care represents the most effective methodological instrument to constantly improve the quality of life and increase the care of patients affected with liver diseases. This health policy responds to a systematized and organized method aimed at providing care, through

229 Vol. 5, núm. 2, abril 2019, pp. 228-251
\end{abstract}


assessment, diagnosis, planning, intervention and evaluation according to the human responses of the person, using the scientific method as a basis for actions, that allows to approach a plan of care, according to the pathology and severity of the patient, considering for that that, the patients with liver disease (liver diseases), chronic, being between them, hepatitis A, B and C, Fatty Liver, Autoimmune Diseases and Cirrhosis; the latter characterized by having a higher risk of hospital readmission, finding a mortality of $13 \%$ at 90 days, and that the most common causes of this medical condition in developed countries are chronic viral hepatitis, B or C, alcoholic liver disease, hemochromatosis and Non-alcoholic liver disease, less common are autoimmune hepatitis, primary and secondary biliary cirrhosis, primary sclerosing cholangitis, medication such as methotrexate and isoniazid, Wilson's disease, alpha-1 antitrypsin deficiency, these conditions which, due to their clinical condition, must be treated under medical guidelines directed by the different programs and policies in health, which have the purpose of guaranteeing the populations (in this case patients with liver diseases) the continuity of the care guaranteeing on a scientific basis, both the evaluation and the application of the appropriate treatment ; hence, the importance of these health care services, which seek to provide patients with chronic liver failure with an opportune diagnosis and the identification of precipitating factors, thus achieving, improve the clinical status of the person.

Key words: Comprehensive Medical Care; Health Policies; Hepatic Diseases; Hepatic Cirrhosis; Hepatic Encephalopathy.

\section{Resumo}

Assistência médica abrangente representa o instrumento metodológico mais eficaz para melhorar constantemente a qualidade de vida e aumentar o atendimento de pacientes afetados por doenças hepáticas. Esta política de saúde responde a um método sistematizado e organizado que visa proporcionar assistência, por meio de avaliação, diagnóstico, planejamento, intervenção e avaliação de acordo com as respostas humanas da pessoa, utilizando o método científico como base para ações, que permite abordar um plano de cuidado, segundo a patologia e gravidade do paciente, considerando para isso que, os pacientes com hepatopatia (hepatopatias), crônicos, estão entre eles, hepatites A, B e C, Fígado Gorduroso, Doenças Auto-Imunes e Cirrose; esta última caracterizada por apresentar maior risco de readmissão hospitalar, encontrando uma mortalidade de 13\% aos 90 dias, e que as causas mais comuns desta condição médica nos países desenvolvidos são hepatite

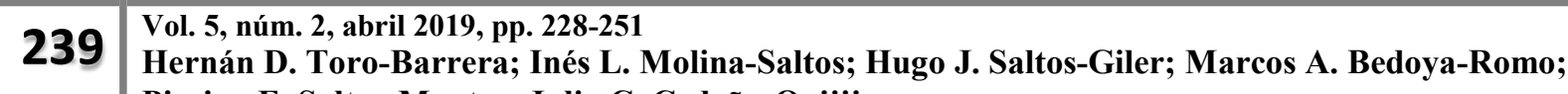


viral crônica, B ou C, hepatopatia alcoólica, hemocromatose e Doenças hepáticas não alcoólicas, menos comuns são hepatite autoimune, cirrose biliar primária e secundária, colangite esclerosante primária, medicação como metotrexato e isoniazida, doença de Wilson, deficiência de alfa-1 antitripsina, estas condições que, devido à sua condição clínica, devem ser tratadas sob diretrizes médicas dirigidas pelos diferentes programas e políticas em saúde, que têm o objetivo de garantir às populações (neste caso pacientes com hepatopatias) a continuidade do cuidado garantindo em bases científicas, tanto a avaliação quanto a aplicação dos cuidados. tratamento adequado; daí, a importância desses serviços de saúde, que buscam proporcionar ao paciente com insuficiência hepática crônica um diagnóstico oportuno e a identificação de fatores precipitantes, conseguindo assim, melhorar o estado clínico da pessoa.

Palavras chave: Assistência Médica Abrangente; Políticas de Saúde; Doenças Hepáticas; Cirrose Hepática; Encefalopatia hepática.

\section{Introducción.}

Los planes de intervención médica representan en la actualidad una medida social que le permite a las personas y comunidades en general minimizar los riesgos de sufrir de las enfermedades de más alta prevalencia, disminuyendo así, el nivel de los factores de riesgo o de la probabilidad de su ocurrencia. Entre sus propósitos, se encuentra orientar a las personas sobre los factores que inciden en la prevalencia u origen de determinada enfermedad o problema de salud pública, con el fin de minimizar aquellas patologías que son de alta ocurrencia poblacional.

Entiéndase así, que estas intervenciones médicas integrales representan un proceso social, cultural, educativo y político que incrementa la conciencia ciudadana sobre la salud y desarrollo, promueve estilos de vida saludables y la movilización social a favor de la salud; genera igualdad de oportunidades y poder a las personas para que ejerzan sus derechos y responsabilidades a partir del desarrollo de políticas, planes, programas y proyectos que sean favorables a su salud y bienestar. Según (Ardila, 2013):

La calidad de vida es un concepto que implica múltiples factores que incluyen tanto lo físico como lo psicológico, y el análisis de las necesidades y motivaciones humanas. Por lo tanto, este concepto debe ser abordado desde diferentes saberes, entre ellos el psicológico, con grandes aportes a esta

\footnotetext{
231 Vol. 5, núm. 2, abril 2019, pp. 228-251 
área del desarrollo social, minimizando así el extremo énfasis de la psicología tradicional, limitada a la solución de déficits, sin contribuir al avance y desarrollo del individuo (p.74).

En razón a esto, se debe mencionar que para el alcance de la calidad de vida fueron establecidas: la asistencia sanitaria ambulatoria, domiciliaria y de urgencias, así como la realización de actividades orientadas a la promoción de la salud y a la prevención de la enfermedad. Autores como (Geroudd, 2014) afirman que la atención médica integral, "centra su propósito en la asistencia sanitaria esencial basada en métodos y tecnologías prácticas”. (p.22). Dichas acciones, son, científicamente fundadas y socialmente aceptables, pues se encuentran al alcance de todos los individuos y familias de la comunidad mediante su plena participación a costos aceptables para cada Nación.

De esta manera, se determina que la Atención Médica Integral, representa el primer nivel de contacto de los pueblos, individuos, la familia y la comunidad con el sistema nacional de salud, llevando lo más cerca posible la atención y nociones en materia de sanación y prevención al lugar donde residen y trabajan las personas, y constituye el primer elemento de un proceso permanente de asistencia sanitaria, el cual se orienta hacia los principales problemas de salud de la comunidad.

En efecto, la atención médica representa un conjunto de medidas y políticas establecidas con el fin de prestar los servicios de promoción, prevención, tratamiento y rehabilitación necesarios para resolver problemas de salud pública como lo son: las enfermedades crónicas no transmisibles, dengue, enfermedades de transmisión sexual y enfermedades hepáticas;estas últimas consideradas como una importante causa de morbilidad y mortalidad, siendo la Hepatitis Viral de alta endemicidad y la Cirrosis Hepática el motivo más frecuente de presentación crónica asociada a mortalidad reflejada en países latinoamericanos.

Por consiguiente, se puede entender que el término enfermedades hepáticas aplica a muchas patologías, siendo las mismas definidas por enfermedades del hígado o anomalías que impiden que el hígado funcione o evitan que trabaje bien, las mismas se caracterizan en el paciente generalmente por presentar dolor abdominal, el color amarillo de la piel o los ojos (ictericia) o los resultados anormales de las pruebas de la función hepática. Para (Wenz, 2013)

Entre las patologías más comunes de este tipo de patología se encuentran las enfermedades causadas por virus, como la hepatitis A, la hepatitis B y la hepatitis C, las enfermedades causadas por drogas,

\footnotetext{
232 Vol. 5, núm. 2, abril 2019, pp. 228-251 
venenos o toxinas o por ingerir demasiado alcohol. Los ejemplos incluyen enfermedad por hígado graso y cirrosis. El Cáncer de hígado y las Enfermedades hereditarias, como hemocromatosis y la enfermedad de Wilson. (p.54)

Lo antes descrito lleva a determinar que las enfermedades hepáticas son aquellas relacionadas con el mal funcionamiento del hígado. Sus síntomas pueden variar, pero a menudo incluyen hinchazón del abdomen y las piernas, tener moretones con facilidad, cambios en el color de las heces y la orina, y piel y ojos amarillentos o ictericia. No obstante, en muchos casos no se presentan síntomas, siendo las pruebas de imagen o de función hepática, las que pueden comprobar si hay daño en el hígado o ayudar a diagnosticar enfermedades del hígado. Del mismo modo, Wenz (Obcit), afirma:

Las enfermedades hepáticas suelen producirse por hepatitis viral, hepatitis A, B y C, enfermedad hepática grasa y cirrosis. El hígado y sus células cambian de manera drástica cuando un hígado normal se vuelve graso o cirrótico. La enfermedad hepática puede ser hereditaria (genética) o causada por diferentes factores que dañan el hígado, como los virus y el consumo de alcohol y la obesidad. Con el tiempo el daño hepático provoca cicatrización (cirrosis) que puede producir insuficiencia hepática, un trastorno que pone en riesgo la vida, de ello, la importancia, de que el paciente con este tipo de patologías conozca sus riesgos y modos de autocuidado. (p.56)

Corresponde entonces señalar que, las enfermedades hepáticas representan un problema de importancia para la salud pública a nivel mundial. La recurrencia e incidencia de estas patologías ocupan el lugar número 14 en el mundo entero. Las causas más frecuentes de daño hepático crónico en países desarrollados son el consumo de alcohol, la infección por virus de la hepatitis c, enfermedad hepática no alcohólica. Asimismo, se observa que, en países subdesarrollados, haciendo mención a gran parte del continente africano, la causa más frecuente de cirrosis hepática es la infección por el virus de la hepatitis b. Asimismo, en México, la cirrosis hepática ocupa el $4^{\circ}$ lugar de mortalidad en la población general, y se estima que para el año 2020 habrá 1496000 cirróticos a nivel nacional en el año 2008, hubo 31528 defunciones atribuidas a cirrosis hepática, siendo en un 75\% (Zett, 2013).

Datos epidemiológicos realizados en el 2017 por la Organización Panamericana de la Salud OPS, demuestran que las enfermedades hepáticas, específicamente la cirrosis son consideradas de

\footnotetext{
233 Vol. 5, núm. 2, abril 2019, pp. 228-251

Hernán D. Toro-Barrera; Inés L. Molina-Saltos; Hugo J. Saltos-Giler; Marcos A. Bedoya-Romo;

Pierina E. Saltos-Montes; Julio C. Cedeño-Quijije
} 
presentación asintomática "hasta que se manifiestan las complicaciones con ictericia, eritema palmar, ascitis, hedor hepático, anorexia y pérdida de peso entre otras”, destacándose entre sus causasprincipalmente el consumo crónico de alcohol y la enfermedad viral crónica (Hepatitis B y C). (p.11).

En el caso de la Cirrosis, se debe destacar que esta patología representael estado final del daño hepatocelular crónico, el cual se caracteriza por necroinflamacion y fibrosis hepática difusa, secundaria a un daño crónico o agudo, con formación de nódulos de regeneración y presencia de una alteración en la morfología de la unidad funcional hepática, aumentando las resistencias vasculares hepáticas, causando finalmente hipertensión portal y alteraciones en la función de síntesis del mismo.

La progresión del daño de esta enfermedad varía de persona a persona. Su pronóstico hasta hace algunos años se relaciona íntimamente con la escala ChildPugh, agrupando el grado de insuficiencia hepática en 3 grupos ( $a, b, c)$ tomando en cuenta las funciones hepatocelulares desde una perspectiva bioquímica (bilirrubinas, síntesis de proteínas) y la presencia de complicaciones de la misma, tales como ascitis, encefalopatía. La sobrevida para la clase a es de $100 \%$ en un año, mientras que aquellos que se encuentren en la clase c tiene una sobrevida de $45 \%$ a un año, lo cual recalca la importancia del diagnóstico y la prevención de las complicaciones en el paciente cirrótico. (Vera, 2013)

En unión de esto, la Organización Mundial de la Salud (OMS, 2017), a través de artículos científicos informativos señala que "la primera causa de cirrosis hepática es el consumo crónico de alcohol o etanol". (p.14). Por ende, se puede entender que el consumo inveterado de alcohol representa un problema social, lo que representa a su vez un deterioro socioeconómico, incremento de la violencia familiar, accidentes de tránsito, etc.

Del mismo modo, se describe la encefalopatía hepática, la cual se encuentra dentro de las principales causas de ingreso a hospitales de segundo nivel por descompensación de cirrosis hepática,junto con la hemorragia de tubo digestivo alto y la peritonitis bacteriana espontanea sintomática. Por ende, la encefalopatía hepática representa clínicamente un trastorno en el cual el diagnostico se realiza por exclusión, siempre y cuando se descarten otras causas de deterioro neurológico agudo. Esta

\footnotetext{
\begin{tabular}{l|l}
234 & Vol. 5, núm. 2, abril 2019, pp. 228-251 \\
Hernán D. Toro-Barrera; Inés L. Molina-Saltos; Hugo J. Saltos-Giler; Marcos A. Bedoya-Romo;
\end{tabular} 
patología, se puede presentar bajo tres contextos, en pacientes portadores de insuficiencia hepática crónica, pacientes portadores de falla hepática aguda y pacientes portadores de cortocircuitos portosistemicos, siendo más frecuente en países occidentales la causa crónica, principalmente secundaria a cirrosis hepática alcohólico nutricional. (Barreiro 2015: 43)

En unión de esto, se presenta la enfermedad grasa hepática no alcohólica (EGHNA), la cual es reconocida como una condición médica. Esta enfermedad se caracteriza por cambios grasos en el hígado, que van desde simple esteatosis a esteatohepatitis, fibrosis avanzada y cirrosis. (Carcett 2014: 21) . La frecuencia de la EGHNA no es bien conocida, pero se han publicado algunos datos que revelan su presencia; en el $20 \%$ de las ecografías abdominales, el $27 \%$ de las biopsias hepáticas, el $20 \%$ de las autopsias y el $25 \%$ de los donantes de hígado para trasplante. (Bestit, 2013).

Por tanto, se calcula que afecta entre $10-24 \%$ de la población general, y su prevalencia aumenta hasta $57-74 \%$ en personas obesas. Aproximadamente el 7,5\% de la población general padece diabetes y se estima que el $50 \%$ de ellos presenta cierto grado deenfermedad hepática grasa no alcohólica. La esteatohepatitis (esteatosis + inflamación) no alcohólica (EHNA), afecta al $3 \%$ de sujetos delgados, $19 \%$ de obesos, y casi la mitad de obesos mórbidos, según lo publicado en series extranjeras. (Bestit: Obcit: 21).

Los señalamientos antes descritos llevan a establecer que las enfermedades hepáticas son reconocidas a nivel mundial como un problema de salud pública, las mismas, a nivel epidemiológico, han demostrado altos índices de morbi - mortalidad. Tales enfermedades reclaman un nivel de autocuidado preventivo, que sólo puede alcanzarse, a través de la promoción de medidas de salud, las cuales logren educar a las poblaciones de pacientes afectados con estas patologías, sobre la forma cómo minimizar complicaciones y la manera cómo desarrollar medidas de autocuidado que permitan alcanzar niveles de vida óptimos; condición ésta, para lo que han sido diseñados los programas de atención médica a la salud. Para (De Santis, 2015):

La atención médica integral implica una serie de eventos sociales y comunitarios, los cuales comprenden acciones encaminadas al desarrollo y liberación del conocimiento preventivo del ser humano. Esta política de salud, involucra todas aquellas actividades asistenciales de atención individual, diagnósticas, terapéuticas y de seguimiento de procesos agudos o crónicos, así como

\footnotetext{
235 Vol. 5, núm. 2, abril 2019, pp. 228-251

Hernán D. Toro-Barrera; Inés L. Molina-Saltos; Hugo J. Saltos-Giler; Marcos A. Bedoya-Romo;

Pierina E. Saltos-Montes; Julio C. Cedeño-Quijije
} 
aquellas de promoción de la salud, educación sanitaria y prevención de la enfermedad que realizan los diferentes profesionales de atención primaria. (p.87)

Lo descrito lleva a comprender que, las políticas y medidas en salud comprenden todas aquellas actividades dirigidas a modificar o potenciar hábitos y actitudes que conduzcan a formas de vida saludable, así como a promover el cambio de conductas relacionadas con factores de riesgo de problemas de salud específicos y las orientadas al fomento de los autocuidados de riesgos de enfermedades como lo son en este caso en específico, las enfermedades hepáticas. De aquí, la importancia del presente artículo científico, el cual centra su atención en analizar la atención médica integral en pacientes con enfermedad hepática.

\section{Método.}

Para el desarrollo de este proceso investigativo, se plantea como metodología la encaminada hacia una orientación científica particular que se encuentra determinada por la necesidad de indagar en forma precisa y coherente una situación, en tal sentido, Dávila (2012), define la metodología “como aquellos pasos previos que son seleccionados por el investigador para lograr resultados favorables que le ayuden a plantear nuevas ideas". (p53.)

Lo citado por el autor, lleva a entender que el desarrollo de la acción investigativa busca simplemente coordinar acciones enmarcadas en una revisión bibliográfica con el fin de complementar ideas previas relacionadas con: "La atención médica integral en pacientes con enfermedad hepática" para así finalmente elaborar un cuerpo de consideraciones generales que ayuden a ampliar el interés propuesto.

\section{Tipo de Investigación}

Dentro de toda práctica investigativa, se precisan acciones de carácter metodológico mediante las cuales, se logra conocer y proyectar los eventos posibles que la determinan, así como las características que hacen del acto científico un proceso interactivo ajustado a una realidad posible de ser interpretada. En este sentido, se puede decir, que la presente investigación corresponde al tipo documental, definido por (Dávila Obcit), "se ocupa del estudio de problemas planteados a nivel

\footnotetext{
236 Vol. 5, núm. 2, abril 2019, pp. 228-251 
teórico, la información requerida para abordarlos se encuentra básicamente en materiales impresos, audiovisuales y /o electrónicos". (p.41).

En consideración a esta definición, la orientación metodológicapermitió la oportunidad de cumplir con una serie de actividades inherentes a la revisión y lectura de diversos documentos donde se encontraron ideas explicitas relacionadas con los tópicos encargados de identificar a cada característica insertada en el estudio. Por lo tanto, se realizaron continuas interpretaciones con el claro propósito de revisar aquellas apreciaciones o investigaciones propuestas por diferentes investigadores relacionadas con el tema de interés, para luego dar la respectiva argumentación a los planteamientos, en función a las necesidades encontradas en la indagación.

\section{Fuentes Documentales}

El análisis correspondiente a las características que predomina en el tema seleccionado, llevan a incluir diferentes fuentes documentales encargadas de darle el respectivo apoyo y en ese sentido cumplir con la valoración de los hechos a fin de generar nuevos criterios que sirven de referencia a otros procesos investigativos. Para (Arias, 2010), las fuentes documentales incorporadas en la investigación documental o bibliográfica, "representa la suma de materiales sistemáticos que son revisados en forma rigurosa y profunda para llegar a un análisis del fenómeno". (p.41). Por lo tanto, se procedió a cumplir con la realización de una lectura previa determinada por encontrar aquellos aspectos estrechamente vinculados con el tema, para luego explicar mediante un desarrollo las respectivas apreciaciones generales de importancia.

La conducción de la investigación para ser realizada en función a las particularidades que determinan a los estudios documentales, tiene como fin el desarrollo de un conjunto de acciones encargadas de llevar a la selección de técnicas estrechamente vinculadas con las características del estudio. En tal sentido, (Arias Obcit) refiere, que es "una técnica particular para aportar ayuda a los procedimientos de selección de las ideas primarias y secundarias". (p. 71).

Por ello, se procedió a la utilización del subrayado, resúmenes, fichaje, como parte básica para la revisión y selección de los documentos que presentan el contenido teórico. Es decir, que mediante su aplicación de estas técnicas se pudo llegar a recoger informaciones en cuanto a la revisión bibliográfica de los diversos elementos encargados de orientar el proceso de investigación. Tal como

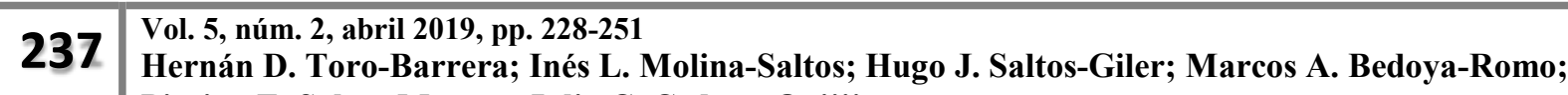


lo expresa, (Arias Obcit) "las técnicas documentales proporcionan las herramientas esenciales y determinantes para responder a los objetivos formulados y llegar a resultados efectivos" (p. 58). Es decir, para responder con eficiencia a las necesidades investigativas, se introdujeron como técnica de recolección el método inductivo, que hizo posible llevar a cabo una valoración de los hechos de forma particular para llegar a la explicación desde una visión general.

Asimismo, se emplearon las técnicas de análisis de información para la realización de la investigación que fue ejecutada bajo la dinámica de aplicar diversos elementos encargados de determinar el camino a recorrer por el estudio, según, (Arias, Obcit) las técnicas de procesamiento de datos en los estudios documentales "son las encargadas de ofrecer al investigador la visión o pasos que debe cumplir durante su ejercicio, cada una de ellas debe estar en correspondencia con el nivel a emplear" (p. 123). Esto indica, que para llevar a cabo el procesamiento de los datos obtenidos una vez aplicado las técnicas seleccionadas, tales como: fichas de resumen, textual, registros descriptivos entre otros, los mismos se deben ajustar al nivel que ha sido seleccionado.

\section{Resultados.}

\section{Atención Médica Integral}

A través de la historia de la humanidad, los pueblos han buscado mantener, proteger y recuperar su salud: para ello, los gobiernos han organizado diferentes políticas con el fin de optimizar los recursos humanos, físicos, económicos y ambientales, partiendo de enfoques y estrategias variadas de acuerdo al momento y situación social que se esté viviendo. He aquí, la importancia de los programas de intervención médica integral establecidos por los gobiernos y las diferentes organizaciones, los cuales buscan concientizar a los habitantes de las diferentes poblaciones sobre las causas y efectos que tienen determinadas patologías médicas con el fin de promocionar la salud.

A tal efecto, hablar de atención médica integral es hacer referencia a la promoción de la salud, reconocida como el conjunto de estrategias médico - comunitarias dirigidas hacia el fomento de estilos de vida saludables, los cuales orientan sus esfuerzos, tanto a la población en general, como a los individuos en el contexto de su vida cotidiana; buscando con ello, actuar en lo colectivo sobre los determinantes de la salud, promoviendo las normas de auto cuidado a seguir y las previsiones médicas necesarias, a fin de alcanzar el bienestar tanto de la persona sana como de la enferma.

\footnotetext{
238 Vol. 5, núm. 2, abril 2019, pp. 228-251 Hernán D. Toro-Barrera; Inés L. Molina-Saltos; Hugo J. Saltos-Giler; Marcos A. Bedoya-Romo; Pierina E. Saltos-Montes; Julio C. Cedeño-Quijije
} 
Bajo este enfoque se debe entender que, la atención médica integral representan una medida social, la cual permite a las personas y comunidades en general minimizar los riesgos de sufrir de las enfermedades de más alta prevalencia, disminuyendo así, el nivel de los factores de riesgo o de la probabilidad de su ocurrencia. Tal consideración, lleva a reflexionar sobre la importancia social que tienen los planes de intervención médicos comunitarios, ya que los mismos se basan en el desarrollo integral de la salud incorporando para ello, programas nacionales apropiados para cada situación o patología a tratarse.

Por ende, laatención médica integral busca desarrollar tanto el potencial del individuo como de su familia, la comunidad, la sociedad y también del medioambiente, entendiendo éste como las circunstancias físicas, interpersonales y económicas en que se desempeña la persona. En otras palabras, es una aproximación proactiva a la salud que incluye el desarrollo de fuerzas, incremento de recursos y fomento de la resiliencia con el fin de lograr el funcionamiento óptimo del ser humano (Ramony, 2016).

De allí, la necesidad de ejecutar políticas públicas que incluyan programas que vayan en aras de contribuir con la reducción de los factores de riesgo y en la pesquisa temprana de enfermedades de alto riesgo, como lo es en este caso en particular las enfermedades de orden hepático. Estos programas tienen como propósito brindar una atención médica integral que permita fortalecer los conocimientos de las personas, modificar actitudes y percepciones en la población y de esta manera favorecer la adopción de estilos de vida saludable en la comunidad.

De esta manera, es de gran importancia que la atención médica integral o programas de salud, se encuentren dirigidos a concientizar a las comunidades sobre la importancia que tiene el desarrollar estilos de vida saludables y, del mismo modo orientar sobre los factores de riesgo que incidan en la proliferación de enfermedades, lográndose a través de ellos, un proceso de enseñanza- aprendizaje que pretenda conducir al individuo y la colectividad a un proceso de cambio en donde se promueva la conservación y mejoramiento de su salud.

En virtud de esto, se hace necesario resaltar que existe la necesidad de intensificar y expandir la atención médica integral en base al desarrollo de programas educativos e investigaciones de orden sanitario, las cuales, a través de gestiones organizadas en salud, busquen reforzar el control

\footnotetext{
\begin{tabular}{l|l}
239 & Vol. 5, núm. 2, abril 2019, pp. 228-251 \\
Hernán D. Toro-Barrera; Inés L. Molina-Saltos; Hugo J. Saltos-Giler; Marcos A. Bedoya-Romo;
\end{tabular} 
adecuado y permanente de los factores y causas que inciden en la prevalencia de este mal, lo cual comprende aspectos específicos tales como el conocimiento acerca de la enfermedad que poseen las personas, los factores que inciden en su aparición y complicaciones médicas, sus riesgos y las múltiples consecuencias que genera; aspecto éste representativo sobre la morbi mortalidad que han generado las enfermedades hepáticas en la sociedad.

\section{Enfermedades Hepáticas}

Las enfermedades hepáticas son reconocidas como las enfermedades del hígado; estas afecciones de la salud provienen del mal funcionamiento de este órgano. Los síntomas de esta enfermedad pueden variar, generalmente es asintomática y se pronuncia en el paciente cuando ya existe alguna complicación; los mismos incluyen hinchazón del abdomen y las piernas, tener moretones con facilidad, cambios en el color de las heces y la orina, y piel y ojos amarillentos o ictericia. En términos generales, el término "enfermedad hepática" puede ser aplicado a muchas enfermedades que impiden que el hígado funcione o evitan que trabaje bien.

\section{Figura $N^{\circ} 1$. Hígado}

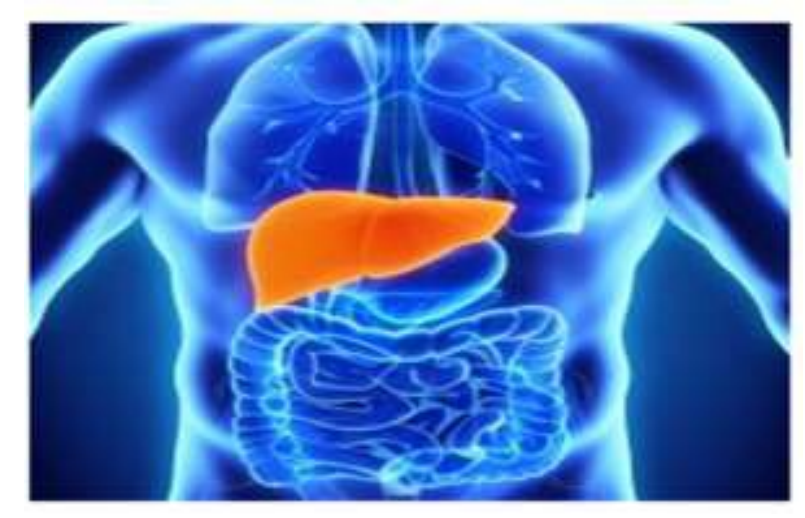

Fuente: Ramony (2016)

El hígado es un órgano que tiene una capacidad funcional enorme, el mismo, contribuye a almacenar, procesar o eliminar todo lo que ingerimos, y así se genera la energía que necesitamos para funcionar diariamente. Este órgano, tiene una cualidad sorprendente: su gran capacidad de regeneración. Si a un paciente se le quita la mitad de su hígado, en tres semanas éste vuelve a crecer, 
y alcanza su volumen anterior. Esto sucede en un hígado sano. Sin embargo, es también por esto que el hígado puede soportar años sin síntomas de daño, aún y cuando éste ya haya ocurrido.

Las enfermedades del hígado son la cuarta causa en países latinoamericanos(Barrera, 2014)Su prevalencia como enfermedad es extraordinariamente grande. A diferencia de la creencia popular, el consumo de alcohol no es necesariamente la causa de un hígado enfermo. Aunque definitivamente esto incide, la enfermedad ocasionada por el virus de la hepatitis $\mathrm{C}$ es también una de las principales causas a nivel mundial de cirrosis.Actualmente el síndrome metabólico, relacionado con la obesidad y el sobrepeso, conlleva a la enfermedad conocida comúnmente como hígado graso. Este padecimiento tiene que ver no sólo con la cantidad de los alimentos que ingerimos sino también con su calidad.

Lo reseñado sobre enfermedades hepáticas lleva a destacar que, el hígado es el órgano de mayor tamaño dentro del cuerpo. Su función se centra en ayudar al organismo a digerir los alimentos, almacenando energía y eliminando toxinas. De allí, que existan muchos tipos de enfermedades hepáticas, destacándose entre ellas:

- Enfermedades causadas por virus, como la hepatitis A, la hepatitis B y la hepatitis C.

- Enfermedades causadas por drogas, venenos o toxinas o por ingerir demasiado alcohol (enfermedad por hígado graso y cirrosis)

- Cáncer de hígado.

- $\quad$ Enfermedades hereditarias, como hemocromatosis y la enfermedad de Wilson.

En efecto, existen muchos tipos de enfermedades hepáticas, siendo algunas de ellas de mayor incidencia y recurrencia observados en los índices de morbilidad y mortalidad presentes en los sistemas sanitarios; lo que en consecuencia lleva a determinar que este tipo de patologías representa un problema de salud pública, el cual para su control amerita de una atención médica integral, la cual mediante políticas, sistemas y servicios de salud logren orientar a los pacientes afectados con este tipo de mal de salud, sobre las medidas médicas a seguir para su cuidado y cuáles son los factores de riesgo asociados.

En virtud de lo descrito, Ramony (2016), en aquí, el surgimiento de infinitos estudios dirigidos a identificar cuáles son los factores de riesgo que más inciden en la aparición de enfermedades 
hepáticas, siendo los resultados más comunes los relacionados con la cirrosis hepática, para este autor:

Estudios recientes realizados en países como Perú y Ecuador que buscan determinar los factores de riesgo asociados a cirrosis hepática se encuentra el alcoholismo como principal factor de riesgo asociado a cirrosis hepático. En Perú, se encuentra la hepatitis B como principal factor de riesgo asociado a cirrosis hepática, esto difiere de lo encontrado en países desarrollados donde la hepatitis $\mathrm{C}$ es la primera causa de cirrosis hepática, y en tercer lugar la hepatitis B, y en otros estudios desarrollados en Ecuador, representa el segundo factor de riesgo asociado a cirrosis hepática, corroborado esto con los estudios de prevalencia de hepatitis $\mathrm{B}$, donde se concluye que la hepatitis B es la que tiene mayor endemicidad entre las hepatitis virales que en el Perú.(p.32).

De la misma forma, Rodríguez en su estudio Factores de riesgo asociados a la cirrosis hepática demostraron lo siguiente:

\section{Gráfico $N^{\circ} 1$ Factores de Riesgo asociados a la Cirrosis Hepática}

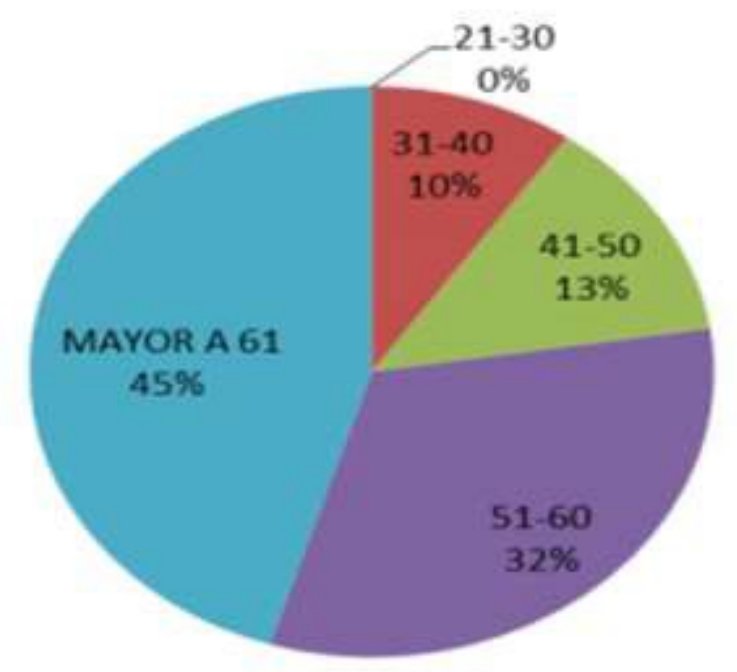

Fuente: Geroudd (2014) 
Los resultados expuestos, permiten demostrar que el alcoholismo representa el principal factor de riesgo que se encuentra asociado a las enfermedades hepáticas y sus complicaciones, siendo la de mayor prevalencia e incidencia la Cirrosis Hepática, presente en mayor número en mayores de 61 años de edad.

Entre las enfermedades hepáticas de mayor recurrencia a nivel sanitario, se encuentran las siguientes:

\section{Cirrosis Hepática}

El consumo excesivo de alcohol se asocia con distintas enfermedades hepáticas, entre ellas la esteatosis hepática, hepatitis alcohólica, y finalmente la cirrosis. Esta última, caracterizada por ser una enfermedad crónica y progresiva del hígado, determinada por la degeneración y destrucción de las células y el tejido hepático. Es considerada una de las principales causas de muerte y ha reportado un incremento estadístico constante en los últimos años en países latimoamericanos, de acuerdo al sistema nacional de salud (SINAIS) la cirrosis y otras enfermedades crónicas del hígado ocupan el tercer lugar dentro de las diez principales causas de mortalidad en la población adulta con una tasa del 40.2. (Barrera 2014: 21).

\section{Figura $N^{\circ} 2$ Cirrosis Hepática}

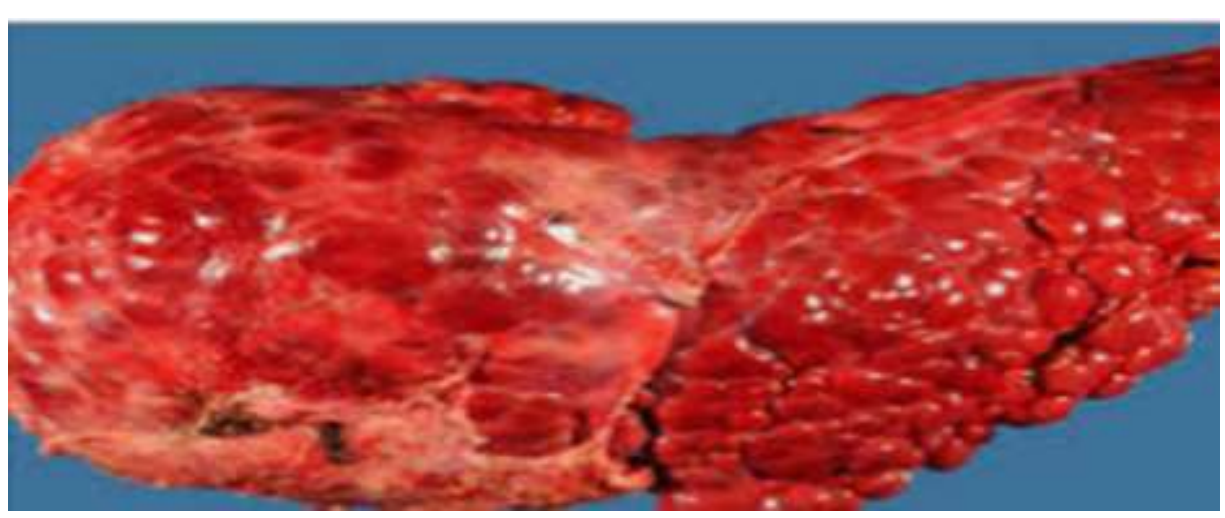

Fuente: Ramony (2016)

Actualmente se ha considerado la cirrosis hepática como uno de los padecimientos de mayor frecuencia en la población adulta, siendo la ingesta de alcohol una de las principales causas de 
enfermedades del hígado. De ahí, que determinar la causa de la cirrosis es de especial importancia para dirigir el tratamiento, algunas clasificaciones morfológicas de la cirrosis han sido abandonadas por sus limitaciones al ser relativamente no especificas con respecto a la etiología, el aspecto del hígado puede cambiar a medida que la enfermedad hepática progresa, tal es así que la cirrosis micronodular, presentación típica cuando la etiología es alcohol, hemocromatosis y colestasis, puede cambiar a macronodular que es la presentación común cuando la etiología es vírica. También existen marcadores serológicos disponibles hoy en día que son más específicos que la morfología hepática para determinar la etiología de la cirrosis. (Barrera Obcit: 22). Unido a esto, (Terranueva, 2016), exponen:

En Ecuador en el año 2015 al revisar 157 historias clínicas buscando factores de riesgo predisponentes asociados a cirrosis hepática se encontró el consumo crónico de alcohol presente en $56.1 \%$ ( 88 pacientes), la hepatitis B y C presente en el $8.9 \%$ (14 pacientes) y $2.5 \%$ (4 pacientes) respectivamente. Las transfusiones antes del diagnóstico de cirrosis hepática se presentó en el 1.3\% ( 2 pacientes) y drogadicción vía nasal en el $0.6 \%$ (1 paciente). En relación al consumo de alcohol se encontró una mediana de 26 años de consumo. Entre las historias no se encontró información de drogadicción endovenosa. (p.54).

En general, se considera la cirrosis como una patología irreversible cuando está en sus etapas avanzadas, momento en el cual la única opción de tratamiento puede ser el trasplante hepático. Sin embargo, se ha documentado reversibilidad en las etapas tempranas de la enfermedad, y siguiendo un tratamiento dirigido a la causa de fondo. La cirrosis representa el estadio tardío de cualquier enfermedad hepática crónica, causando tanto inflamación hepática crónica o colestasis.

\section{Encefalopatía Hepática}

La encefalopatía hepática es una de las principales manifestaciones de descompensación en el paciente portador de insuficiencia hepática crónica, lo cual conlleva a un aumento de la mortalidad de la misma, así como un aumento en el índice de ingresos a hospitales de segundo nivel. Existen diversos factores desencadenantes de encefalopatía hepática, entre los cuales se encuentran las infecciones bacterianas, la cuales tienen un rol importante en la descompensación de pacientes con cirrosis hepática. (Sagastegui, 2013). 
Las más frecuentemente señaladas son la peritonitis bacteriana espontánea y la infección de vías urinarias la cual hasta en un $40 \%$ de los pacientes se encuentra de manera asintomática. Esto se encuentra facilitado por las alteraciones en el estado inmune del paciente cirrótico; no obstante, la infección de vías urinarias específicamente hablando es una entidad poco estudiada como factor precipitante de encefalopatía hepática en pacientes con cirrosis hepática, o como infección concomitante, a pesar de la alta frecuencia de este tipo de infecciones en pacientes cirróticos así como en la población en general (Sagastegui 2013: 21).

Son pocos los estudios encontrados en la literatura que evalúen la prevalencia, prevención y medidas encaminadas a la disminución de este tipo de infecciones específicamente dentro de los pacientes diagnosticados con encefalopatía hepática por lo tanto se pretenderá mostrar la relación que existe entre la presencia de infección de vías urinarias en pacientes con diagnóstico de encefalopatía hepática, así como la relación que existe entre el tratamiento de la misma con la remisión del déficit neurológico, con el fin de que se puedan evaluar posteriormente estrategias profilácticas de infecciones de vías urinarias en el paciente cirrótico.

Anatomopatologicamente, la encefalopatía hepática tiene características especiales, entre ellas se encuentra la presencia de astrocitos tipo Alzheimer II, los cuales tienen núcleos grandes con escasa cromatina y un nucléolo prominente. Estos son localizados en la sustancia gris, en el globo pálido, núcleo estriado, tálamo, oliva inferior y glía de bergmann. Otra característica esencial de la encefalopatía hepática es la presencia de astrocitosedematizados, esto Protocolo de investigación. Febrero 2014 secundario al desequilibrio en el estado osmolar del mismo, sin embargo esta característica no es específica pues puede encontrarse además en otros trastornos como trauma, estados epilépticos y otras encefalopatías. (Sagastegui 2013: 21)

\section{Hepatitis B $y$ C}

La hepatitis C es ocasionada por el llamado "virus de hepatitis C". Existen casos de pacientes que se han contagiado por transfusiones de sangre realizadas entre 1985 y 1995, década en la cual se descubrió el virus. Es muy probable que estos pacientes hayan pasado un largo periodo de su vida, quizá desde su juventud, hasta la madurez, sin haber mostrado un solo síntoma de daño hepático, y estas manifestaciones aparecen una vez que el hígado está irreversiblemente dañado. Por otra parte, 
se encuentra la hepatitis B es otra de las enfermedades crónicas del hígado, causada también por un virus. A diferencia de la hepatitis $\mathrm{C}$, que se transmite por transfusiones, la vía de contagio de la hepatitis B puede ser el contacto sexual o la inhalación de sustancias.Aproximadamente 30 por ciento de estos pacientes podrán desarrollar inflamación crónica, e incluso riesgo de cáncer de hígado. Necesitamos comprender que es una enfermedad detectable, de ahí la importancia de la realización de pruebas en caso de formar parte de un grupo de riesgo.

\section{Enfermedad de Higado Graso}

Las causas de la EGHNA aún son pobremente entendidas, se postula que tiene un origen multifactorial en el que se suman alteraciones metabólicas múltiples con cierta predisposición genética y una evidente influencia ambiental. Del mismo modo, se ha demostrado que los pacientes con EGHNA presentan con frecuencia elementos del síndrome metabólico como obesidad abdominal, alteraciones de la tolerancia a la glucosa, hipertrigliceridemia e hipertensión arterial, y que el riesgo de esteatosis aumenta cuando se asocia a un mayor número de componentes de este síndrome. También algunos autores hanencontrado una alta prevalencia de intolerancia a la glucosa, insulinoresistencia y diabetes en pacientes con EGHNA y su presencia aumentaría el riesgo de progresión a fibrosis.

Por ende, es bien conocida la asociación entre la EGHNA y la obesidad, la diabetes mellitus (DM) y la dislipidemia. Sin embargo, también se ha encontrado EGHNA en pacientes sin éstos factores de riesgo. Recientes estudios sugieren que la hiperinsulinemia y la resistencia a la insulina juegan roles patogénicos en pacientes con EGHNA. Sagastegui 2013: 21) De hecho, se ha reportado una fuerte asociación entre el EGHNA y el síndrome metabólico (SM), definido en el 2005 por la American HeartAssociation y por el NationalHeart, Lung, and BloodInstitute. Según AdultTreatment Panel III, se ha pronunciado que el síndrome metabólico comprende por características: la presencia de 3 ó más de los siguientes criterios:

- Incremento del perímetro de la cintura: $102 \mathrm{~cm}$ en los varones y $88 \mathrm{~cm}$ en las mujeres.

- Elevación de los triglicéridos (TGC): $150 \mathrm{mg} / \mathrm{dl}$, o tratamiento farmacológico por elevación de los triglicéridos. 
- Disminución de las Lipoproteínas de alta Densidad 12 (HDLcolesterol): 40 mg/dl en los varones, $50 \mathrm{mg} / \mathrm{dl}$ en las mujeres, o tratamiento farmacológico para aumentar las concentraciones de HDL colesterol.

- $\quad$ Elevación de la presión arterial: $130 \mathrm{mmHg}$ la sistólica y $85 \mathrm{mmHg}$ la diastólica, o bien tratamiento medicamentoso de la hipertensión.

- Elevación de la glucemia en ayunas: $100 \mathrm{mg} / \mathrm{dl}$ o tratamiento farmacológico de la hiperglucemia. Los pacientes con SM tienen un mayor riesgo de desarrollar enfermedad cardiovascular y DM.

Demuéstrese a través de estos señalamientos que, los pacientes que tienen un gran perímetro abdominal (sobrepeso), elevación de los triglicéridos, colesterol alto, elevación de la glucemia o alta presión arterial, aumentan su riesgo de enfermedad hepática.

\section{Atención Médica Integral en enfermedades Hepáticas}

Efectivamente, debido a la enorme incidencia de enfermedades de orden hepática las autoridades sanitarias de cada Nación se ha visto en la necesidad de implementar programas y políticas de atención médica integral, a través de las cuales se ha buscado orientar a las poblaciones sobre los posibles síntomas y causas que este tipo de patologías origina en la persona, buscándose con ello, educar a las poblaciones, a fin de que las mismas logren concientizar sobre aquellos factores que inciden en la recurrencia de este mal de salud y de esa forma puedan prevenirlos y modificar sus estilos de vida. Según Programa sanitario de Salud efectuado en Instituciones Educativas de la Habana Cuba, existen percepciones y características médicas asociadas con estas enfermedades que indican la prevalencia de este tipo de patologías, encontrándose en sus resultados el nivel de conocimientos que los grupos estudiantiles de diferentes niveles educativos demostraron:

\footnotetext{
\begin{tabular}{l|l|}
247 & Vol. 5, núm. 2, abril 2019, pp. 228-251 \\
Hernán D. Toro-Barrera; Inés L. Mot
\end{tabular} Hernán D. Toro-Barrera; Inés L. Molina-Saltos; Hugo J. Saltos-Giler; Marcos A. Bedoya-Romo; Pierina E. Saltos-Montes; Julio C. Cedeño-Quijije
} 


\section{Cuadro $N^{\circ}$ 1. Percepciones sobre enfermedad Hepática}

\begin{tabular}{|l|c|c|c|c|}
\hline & \multicolumn{4}{|c|}{ Grado de Instrucción } \\
\hline Sintomas ascciados & Primaria & Secundaria & Superior N. U. & Superior U. \\
\hline mal aliento & $23(85.2 \%)$ & $89(71.2 \%)$ & $10(50 \%)$ & $120(55 \%)$ \\
\hline sabor amargo en la boca & $23(85.2 \%)$ & $87(69.6 \%)$ & $12(60 \%)$ & $140(64.2 \%)$ \\
\hline manchas en la cara & $15(55.6 \%)$ & $62(49.6 \%)$ & $10(50 \%)$ & $121(55.5 \%)$ \\
\hline manos que se pelan & $15(55.6 \%)$ & $57(45.6 \%)$ & $11(55 \%)$ & $104(47.7 \%)$ \\
\hline manchas blancas en las unflas & $4(14.8 \%)$ & $14(11.2 \%)$ & $1(5 \%)$ & $16(7.3 \%)$ \\
\hline persona colérica & $19(70.4 \%)$ & $54(43.2 \%)$ & $9(45 \%)$ & $58(26.6 \%)$ \\
\hline comer comidas ricas en grasas & $26(96.3 \%)$ & $115(92 \%)$ & $19(95 \%)$ & $194(89 \%)$ \\
\hline Vinagrera y acidez & $17(63 \%)$ & $68(54.4 \%)$ & $10(50 \%)$ & $84(38.5 \%)$ \\
\hline
\end{tabular}

Fuente: Sistema de Salud de la Habana Cuba (2017)

Asimismo, se plantean los resultados arrojados por el Programa sanitario de Salud efectuado en Instituciones Educativas de la Habana Cuba, ante estudios realizados a las poblaciones estudiantiles de estudiantes de diferentes niveles educativos, a fin de verificar si éstos poseen conocimientos sobre las enfermedades de orden hepático, encontrándose que:

\section{Cuadro $\mathrm{N}^{\circ}$ 2. Conocimientos sobre enfermedades Hepáticas}

\begin{tabular}{|l|c|c|c|c|}
\hline Sintomas & Primaria & Secundaria & Superior N. U. & Superior U. \\
\hline pies/piernas hinchadas & $11(24 \%) 1$ & $21(12 \%)$ & $2(9 \%)$ & $14(4 \%)$ \\
\hline aumento de tamaño del abdomen & $6(13 \%)$ & $30(17 \%)$ & 0 & $42(13 \%)$ \\
\hline qjos amarillos & $19(42 \%)$ & $76(44 \%)$ & $15(68 \%)$ & $176(55 \%)$ \\
\hline prurito & $4(9 \%)$ & $5(3 \%)$ & 0 & $14(4 \%)$ \\
\hline manos que se pelan & $5(11 \%)$ & $42(24 \%)$ & $5(23 \%)$ & $75(23 \%)$ \\
\hline
\end{tabular}

Fuente: Sistema de Salud de la Habana Cuba (2017)

Cifras como estas llevan a establecer que, la puesta en práctica de investigaciones y programas sobre las enfermedades hepáticas, constituyen una herramienta del sistema sanitario y gestión en 
salud pública de gran valor, puesto que representa un componente fundamental para la promoción de medidas preventivas dirigidas a establecer la transmisión de conocimientos y de informaciones necesarias para fomentar en las poblaciones una conciencia preventiva que les permita a los habitantes en general, minimizar los riesgos patologías que como éstas lleguen a ser de orden crónico, afectando de manera considerable su salud y la calidad de vida de los pacientes y familiares en general.

Ante esta posibilidad, es especialmente importante exista la evaluación de las consecuencias de las patologías crónicas, tales como la hepatitis en todas sus especificaciones, la cirrosis hepática y otras enfermedades de este orden; esto, debido a que las medidas fisiológicas aportan datos fundamentales para los clínicos, pero son de interés limitados para los enfermos, quienes precisan satisfacer otras expectativas sobre su enfermedad; necesidad ésta, que en respuesta, lleva a considerar la importancia que tienen la atención médica integral y los planes o programas de intervención de la salud, los cuales están dirigidos a orientar, brindándole a las personas las nociones necesarias, en cuanto al tema de salud se refiere y temas de prevención de complicaciones en patologías de alta prevalencia, como lo son las enfermedades hepáticas y, de aquellos males de la salud, que son generados como complicación de esta condición.

\section{Conclusiones.}

El aumento progresivo de enfermedades crónicas, como la cirrosis, hepatitis en todas sus clasificaciones, encefalpatía hepática, enfermedades del higado graso y otras complicaciones de orden hepático motivó el surgimiento y activación de programas de atención médica integral, los cuales nacen, a través de la puesta en práctica de políticas nacionales de salud en Suramérica dirigidas a motivar la participación de la comunidad en la búsqueda de erradicar aquellas patologías de mayor incidencia y recurrencia clínica, con el propósito de promover lacalidad de vida de las personas.

Estos programas de atención médica integral, o medidas de prevención tienen como propósito influir en el estilo de vida de los ciudadanos, con el fin de minimizar aquellas patologías que son de alta prevalencia; como es el caso de las enfermedades hepáticas, las cuales constituyen un problema creciente a nivel mundial y se caracterizan por ser de larga duración y por lo general evolucionan

\footnotetext{
249 Vol. 5, núm. 2, abril 2019, pp. 228-251 
lentamente.Su origen y evolución se debe a diversos factores de riesgo, muchos de los cuales están asociados a estilos de vida no apropiados, hábitos y costumbres que introduce la modernización, tales como alcoholismo, tabaquismo, dietas inadecuadas y drogas.

Por consiguiente, es importante comprender que, los daños en salud atribuibles a estos factores se pueden disminuir al aplicar técnicas de promoción de estilos de vida saludable y planes en los que se logre concientizar a las poblaciones sobre los factores de riesgo que inciden sobre este tipo de patologías y las posibles complicaciones que de ellas se pueden originar; entiéndase con esto que, este tipo de acciones deben estar dirigidas de forma planificada hacia las comunidades con el fin de que las mismas logren canalizar las informaciones allí recibidas y de esa forma, alcanzar una conciencia personal, la cual permitirá a cada individuo, organizar todos esos factores de riesgo que inciden de manera directa sobre su salud, logrando así mejorar su estilo de vida.

\section{Bibliografía.}

Ardila. (2013). Normas de Salud yEstilos para una vida Saludable: Una vía para la Salud Integral. México:: Editorial Pax.

Arias, F. (2010). Diseño de campo y Tipos de Investigación en Proyectos Científicos. España: Editorial: Luces.

Barrera, P. (2014). Factores de riesgo y Medidas de Prevención de la Cirrosis Hepática. Caracas: Panapo.

Bestit, O. (2013). Enfermedad del Hígado Graso Y SUS Complicaciones Médicas. Pereira, Colombia: Editorial: Las Fuentes.

Dávila, J. (2012). Métodos, enfoques y Paradigmas de la Investigación Científica. Caracas : Editorial Panapo.

De Santis, J. (2015). Programas de atención a la Salud Preventiva. Artículo de Salud de la Escuela de Medicina de la Universidad del Zulia LUZ, en Maracaibo, 8.

Geroudd, C. (2014). Enfermedades Hepáticas y sus complicaciones Médicas. México: Editorial Limusa. .

OMS, O. M. (2017). Complicaciones Médicas de las Enfermedades Hepáticas. Revista Médica de la Universidad de la Salud en la ciudad de México, 16.

\footnotetext{
\begin{tabular}{l|l}
250 & Vol. 5, núm. 2, abril 2019, pp. 228-251 \\
Hernán D. Toro-Barrera; Inés L. Molina-Saltos; Hugo J. Saltos-Giler; Marcos A. Bedoya-Romo;
\end{tabular} Pierina E. Saltos-Montes; Julio C. Cedeño-Quijije
} 
Ramony, V. (2016). Epidemiología Médica de la Cirrosis Hepática. Buenos Aires: Editorial Planeta.

Sagastegui, J. (2013). Encefalopatía Hepática. Revista Factor Salud de la Universidad Autónoma de México en México, 11.

Terranueva, C. y. (2016). Enfermedades Hepáticas y sus complicaciones Médicas. Revista Salud Médica Integral de la Universidad de Los Andes en Mérida Venezuela, 14.

Vera, R. (2013). Enfermedades Hepáticas. Problema de salud Pública. Review of the Epidemiology, 12.

Wenz, G. (2013). Complicaciones Médicas de la Cirrosis Hepática. México: México: 2a ed. Ed.McGraw Hill-Interamericana.

Zett, A. y. Lantz, C. (2013). Características de la Cirrosis Hepática, Medidas Preventivas de esta enfermedad. Revista de Salud Panamericana, 17. 